\title{
Anti-coagulation for Patients with Acute Coronary Syndrome
}

\author{
Atsushi Hirayama
}

\begin{abstract}
Although the anti-platelet therapy is a standard for a patient with acute coronary syndrome with or without coronary intervention, oral anticoagulant has not been used for long time. Several trials demonstrated the beneficial effects on the prevention of reinfarction or stroke, but the risk of serious bleeding increased. Furthermore, good adherence and control of oral anticoagulant were necessary to improve the clinical outcome. So, oral anticoagulant has not been used for the patients with acute coronary syndrome; however, direct thrombin inhibitor or direct anti-Xa have been proven to be equivalent or even better to warfarin in stroke prevention for patients with atrial fibrillation (AF). These new oral anticoagulant (NOAC) has been tested in patients with acute coronary syndrome, and showed the normal dose of NOAC for stroke prevention with dual antiplatelet increased the bleeding risk, but reduced dose of NOAC might be beneficial for the secondary prevention. This combination of anticoagulant and DAPT is especially interested, because of the anti-thrombotic regimen for patients with $A F$ and coronary heart disease who are treated by stent. Triple therapy (warfarin and DAPT) increased bleeding complication and major cardiovascular events compared to dual therapy (warfarin and single anti-platelet) in stable coronary artery disease; however, no trials in acute coronary syndrome are available right now. Furthermore, no data are available in NOAC. Until then, the appropriate combination of NOAC and anti-platelet would be chosen by the physician who assessed the risk of bleeding and thrombosis for each patient.
\end{abstract}

KEY WORDS: acute coronary syndrome, oral anti-coagulant, anti-platelet, stent

\section{Introduction}

Coronary plaque rupture following thrombus formation is a cause of acute coronary syndrome, which is a major determinant of patients with ischemic heart disease. In late 1980, the combination of thrombolytic agent and antiplatelet proved to decrease the short-term mortality in patients with ST-segment elevation myocardial infarction (STEMI), ${ }^{1)}$ and routinely performed and became popular. This strategy was applied to the patients with non STEMI or unstable angina (UA), but failed to approve the beneficial effect on the clinical outcome. In TIMI IIIB trial, ${ }^{2)}$ thrombolytic agent is not beneficial and may be harmful in patients with non Q-wave myocardial infarction (NQMI) and UA. This result implicated that the underlying thrombosis mechanism in STEMI is different from that in NSTEMI and UA. Coronary angioscopic finding revealed that the red or mixed thrombus and white thrombus were observed at the culprit lesion in STEMI and NSTEMI/UA, respectively. ${ }^{3)}$ After the failed thrombolytic agent era, anti-platelet agents were tested for the patients with NSTEMI/UA. In the late 1990, several glycoprotein (GP) IIb/

Division of Cardiology, Department of Medicine, Nihon University School of Medicine, 30-1 Ohyaguchi-kamicho, Itabashi-ku, Tokyo, 173 8610, Japan

doi: $10.7793 /$ jcoron. 21.035
IIIa antagonist were developed and tested in patients with UA and NSTEMI. GP IIb/IIIa receptor blocker abciximab did not improve the outcome in GUSTO IV-ACS, ${ }^{4)}$ but in nearly all studies involving the small-molecule GP IIb/IIIa inhibitors (eptifibatide $^{5)}$ and tirofiban ${ }^{6}$ ), the rates of death and MI were reduced in the treatment groups compared with the comparator group ; however, the difference was not always statistically significant. In all of the trials, the risk of bleeding was greater in the groups receiving a GP IIb/IIIa inhibitor than in the comparator group, but the difference was not always statistically significant. ${ }^{7)}$ These trials suggested the antiplatelet might be the key drug to prevent the myocardial infarction in patients with UA. Another anti-platelet inhibitor, P2Y12 receptor blocker clopidogrel is a next candidate for preventing myocardial infarction. Clopidogrel in Unstable Angina to Prevent Recurrent Events (CURE) trial ${ }^{8)}$ randomized 12,562 patients with UA/NSTEMI to either dual antiplatelet therapy with aspirin plus clopidogrel or to aspirin alone immediately upon presentation. At a mean follow-up of 9 months, dual antiplatelet therapy was associated with a significant reduction in the composite primary endpoint of cardiovascular mortality, nonfatal MI, or stroke, largely due to fewer myocardial infarction. As clopidogrel has been proved to prevent cerebro cardio vascular event significantly than aspirin, clopidogrel become a standard anti-platelet agent in patients 
with ischemic heart disease.

In addition to the anti-platelet agent, coronary intervention has dramatically changed the clinical outcome in patients with acute coronary syndrome. At the same time as the beginning of thrombolysis, primary coronary angioplasty using balloon to the occluded culprit lesion showed highly successful reperfusion. After many clinical trials comparing primary angioplasty and thrombolysis, meta-analysis revealed that primary angioplasty improved the clinical outcome including death. ${ }^{9)}$ Furthermore, the introduction of stent decreased the ischemic events after intervention without mortality reduction; however, the stent thrombosis with low incidence was a major problem. Several major clinical trials to prevent the stent thrombosis demonstrated that dual anti-platelet therapy (DAPT), the combination of aspirin and $\mathrm{P} 2 \mathrm{Y} 12$ receptor antagonist, is the most effective agents to achieve the prevention of stent thrombosis. ${ }^{10)}$ In the recent survey in our country, ${ }^{11)}$ among patients with acute coronary syndrome almost $90 \%$ patients received stent and DAPT, and reflects the good clinical outcome. Therefore, anti-platelet agents have been recognized as the standard regime for the patients with coronary artery disease including acute coronary syndrome. ${ }^{12)}$ The real world registry, J-CAD ${ }^{13)}$ showed the anti-platelet agent and statin improve the clinical outcome after adjusting several factors.

\section{Anti-coagulants for the patients with acute coronary syndrome}

Although the platelet adherence and aggregation following the plaque rupture is the initial step and formed the rigid platelet thrombus, thrombin is also produced at the culprit site. Tissue factors produced by the macrophage in the unstable plaque activate the coagulant cascade and produce thrombin, which also activate platelet aggregation and form the fibrin. The activation and coagulation cascade accelerate the thrombus formation at the culprit site and resulted in the narrowing or occlusion of the coronary vessels. In the acute phase, unfractionated heparin or low molecular heparin is widely used for patients with UA / NSTEMI $^{12)}$ or fibrinolytic agents for patients with STEMI are also used routinely. Prothrombin fragment F1+2 is significantly increased in patients with acute coronary syndrome compared to the patients with stable coronary artery in the acute phase as well as 6 months after the onset. ${ }^{14)}$ This finding suggests that the coagulation cascade is activated continuously even in the chronic phase. Following this evidence, a number of clinical trials have assessed the safety and efficacy of oral anticoagulant agents administered to patients with acute coronary syndrome. In comparison with placebo, oral anticoagulants reduced the incidence of death, reinfarction, and stroke, compared to placebo and compared to aspirin. In WARIS II, ${ }^{15)}$ patients with acute coronary syndrome were randomized to warfarin, aspirin and both and showed that warfarin, in combination with aspirin or given alone, was superior to aspirin alone in the incidence of composite endpoint, death, non fetal myocardial infarction or thrombotic cerebral stroke, but with the increase risk of bleeding. A meta-analysis of the use of warfarin in patients with acute coronary syndrome demonstrated that the warfarin or warfarin plus aspirin were superior to aspirin alone in terms of the prevention of reinfarction, but increased the incidence of major bleeding. ${ }^{16)}$ In addition, the discontinuation of oral anticoagulant as well as oral anticoagulant plus aspirin was significantly higher compared to aspirin alone in ASPECT II trial ${ }^{17)}$ and only patients who were compliant with oral anticoagulant over $70 \%$ of the time derive benefit from its administration. ${ }^{18}$

These results suggested that oral anticoagulant is useful for the secondary prevention in patients with acute coronary syndrome, but the retention of oral anticoagulant to the patients and keeping the good control are difficult in the real world. Aspirin alone or dual antiplatelet is widely used and become a standard care.

\section{New era of oral anticoagulant}

Nobel oral anticoagulant became available in 2009. First of all, dabigatran showed the superiority or non-inferiority to VKA (Vitamin K antagonist) in terms of the prevention of stroke or systemic thrombo-embolism in patients with atrial fibrillation. ${ }^{19)}$ Three kinds of anti-Xa antagonist has become available and showed the similar effects to dabigatran in clinical trials. A meta-analysis showed that NOAC had a favorable risk-benefit profile, with significant reductions in stroke, intracranial hemorrhage, and mortality, and with similar major bleeding as for warfarin, but increased gastrointestinal bleeding. ${ }^{20)}$ In these major clinical trials, prothrombin time-international normalized ratio (PT-INR) was achieved range of 2.0-3.0 with warfarin. The evalutation of warfarin control is assessed by time in therapeutic range (TTR). TTR should be achieved over $70 \%$ to obtain the maximum protection against thromboembolism in $\mathrm{AF}^{21)}$ It will be difficult to achieve over $70 \%$ of TTR in practice, over $60 \%$ of TTR might be enough to prevent ischemic stroke. Four major clinical trials showed the racial difference in the onset of stroke or systemic thromboembolism and the intracranial hemorrhage under the warfarin control using PT-INR of range 2.0-3.0. ${ }^{22,23}$ Figure 1 and 2 showed the relationship between center PT-INR and annual incidence of stroke or systemic thromboembolism and intracranial hemorrhage in 4 clinical studied, respectively. There is a negative correlation between center TTR and the incidence of stroke/systemic thromboembolism both in Asians and non-Asians ; however the slope in non-Asians is steeper than that in Asians and the incidence of stroke/systemic thromboembolism is much higher in Asians compared to the non-Asians 


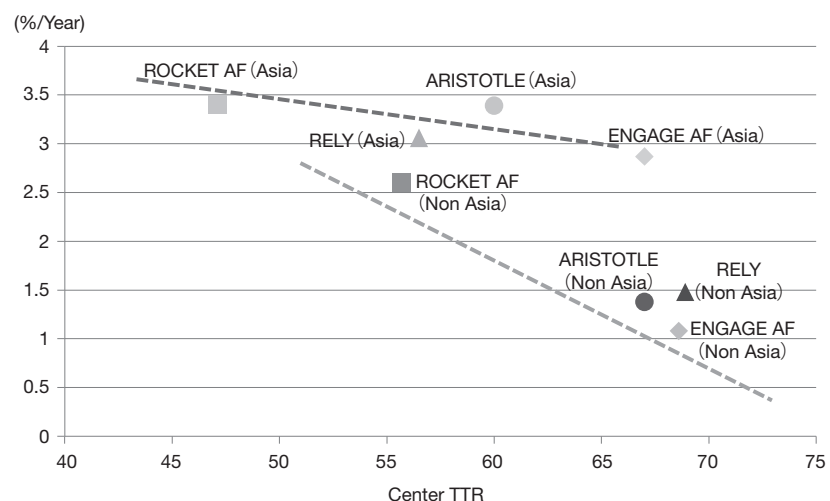

Fig. 1 The relationship between the incidence of stroke/systemic thromboenmolism and center TTR in 4 major clinical trials. The extrapolated line showed the relationship between the incidence of stroke/systemic thromboembolism and center TTR in Asians and nonAsians.

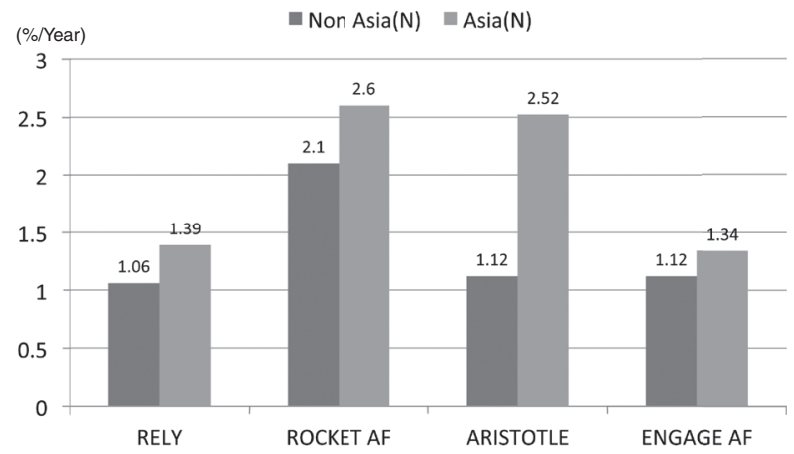

Fig. 3 Incidence of stroke/systemic and thromboembolism in each clinical trials in Asians and non-Asians.

(Fig. 1). These results indicated the higher incidence in Asians but its preventive effect is weaker in Asians. On the other hand, there were no correlation between the incidence of intracranial hemorrhage and center TTR in both Asians and non-Asians, and the incidence of ICH is about 3 or 4 times higher than in nonAsians (Fig. 2). These results demonstrated that ICH occurs in spite of the good control of warfarin, and the prospective study confirms the previous epidemic data; the ICH incidence is 4 times higher than in Caucasian under warfarin control. ${ }^{24)}$ Figure 3 and 4 showed the incidence of stroke/systemic thromboembolism and ICH in NOAC of each studies, respectively. Although the both incidences are slightly higher in Asians than in nonAsians, but the difference is not remarkable compared to warfarin. The reason for the differences between NOAC and warfarin in terms of race is not clear. Genetic back ground or life style including food might explain the difference; however, NOAC is more beneficial in Asians rather than non-Asians.

\section{NOAC in acute coronary syndrome}

Among the clinical trials for prevention of stroke in patients

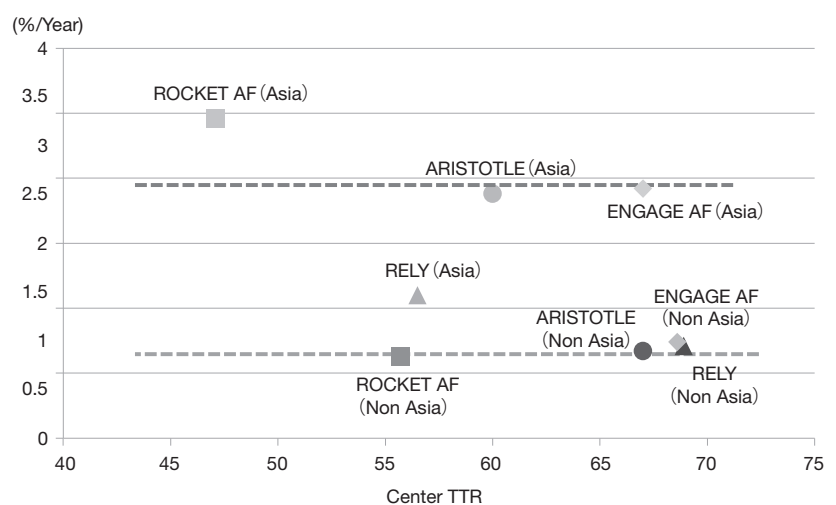

Fig. 2 The relationship between the incidence of intracranial hemorrhage and center TTR in 4 major clinical trials. The extrapolated line showed the relationship between intracranial hemorrhage and center TTR in Asians and non-Asians.

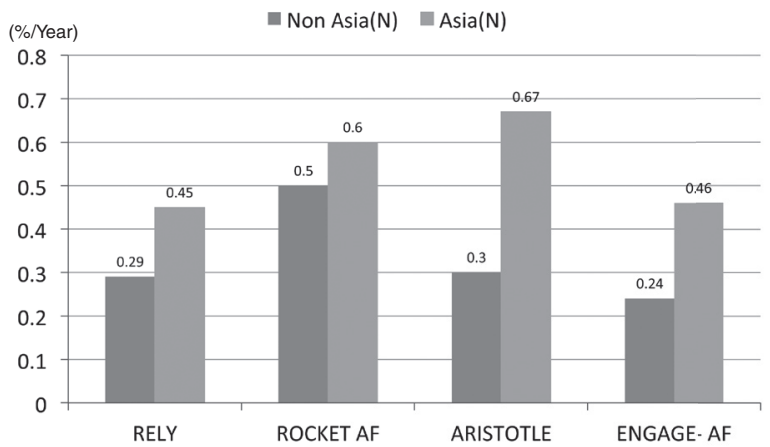

Fig. 4 Incidence of intracranial hemorrhage in each clinical trials in Asians and non-Asians.

with AF, apixaban and rivaroxaban were studied in patients after acute coronary syndrome for the secondary prevention. A randomized, double-blind, placebo-controlled clinical trial comparing apixaban, at a dose of $5 \mathrm{mg}$ twice daily, with placebo, in addition to standard antiplatelet therapy, in high risk patients with a recent acute coronary syndrome for recurrent ischemic events was conducted(APPREASE II) ${ }^{25)}$ This trial was terminated prematurely after recruitment of 7,392 patients because of an increase in major bleeding events with apixaban in the absence of a counterbalancing reduction in recurrent ischemic events. Thus, the addition of apixaban, at a dose of $5 \mathrm{mg}$ twice daily, to antiplatelet therapy in high-risk patients after an acute coronary syndrome increased the number of major bleeding events without a significant reduction in recurrent ischemic events. Apixaban, at a dose of $5 \mathrm{mg}$ twice daily, or placebo was administrated to patients with a recent acute coronary syndrome and at high risk and factors for recurrent ischemic events. The preventive effect of recurrent cardiovascular events of rivaroxaban was also tested in patients with recent acute coronary syndrome. ${ }^{26)}$ In this double-blind, placebo-controlled trial, patients with a recent acute 
coronary syndrome was assigned to receive twice-daily doses of either $2.5 \mathrm{mg}$ or $5 \mathrm{mg}$ of rivaroxaban or placebo for a mean of 13 months and up to 31 months. Rivaroxaban reduced the risk of the composite endpoint of death from cardiovascular causes, myocardial infarction, or stroke (hazard ratio in the rivaroxaban group, $0.84 ; 95 \%$ confidence interval [CI], 0.74 to 0.96 ; $\mathrm{p}=0.008$ ), and increased the risk of major bleeding and intracranial hemorrhage but not the risk of fatal bleeding. In this trial, the twice-daily $2.5 \mathrm{mg}$ dose of rivaroxaban reduced the rates of death from cardiovascular causes $(2.7 \%$ vs. $4.1 \%, \mathrm{p}=0.002)$ and from any cause $(2.9 \%$ vs. $4.5 \%, \mathrm{p}=0.002)$, a survival benefit that was not seen with the twice-daily $5 \mathrm{mg}$ dose. The increased dose of anticoagulant increased the risk of bleeding and resulted in the worse clinical outcome. A meta-analysis including these two large controlled randomized trial showed that the NOAC with a single antiplatelet decreased the risk of ischemic events with the increased risk of bleeding, ${ }^{27)}$ but standard NOAC for the prevention of stroke in AF with DAPT did not have any benefit of the reduction of recurrent cardiovascular events with the increased risk of major bleeding.

\section{V.Anti-thrombotic therapy for the patients with coronary artery disease having NVAF}

Triple therapy, anticoagulant and DAPT, might be the standard for patients with coronary artery disease who are treated with PCI using drug eluting stent (DES) or bear metal stent (BMS) ; however, the risk of serious bleeding increases remarkably as shown in many studies, including BAT study. ${ }^{28)}$ In WOEST tri$\mathrm{al}^{29)} 573$ patients with anticoagulant therapy followed by percutaneous coronary intervention were randomized to either triple therapy (warfarin with DAPT) or double therapy (warfarin and clopidogrel). During 1 year follow-up, bleeding episodes were seen in $54(19.4 \%)$ patients receiving double therapy and in 126 $(44.4 \%)$ receiving triple therapy (hazard ratio [HR] 0.36, 95\% CI $0.26-0.50, \mathrm{p}<0.0001)$. Cumulative incidence of the secondary endpoint (death, myocardial infarction, stroke, target-vessel revascularization, and stent thrombosis) was observed significantly higher in the triple therapy group $(17.6 \%)$ than in the double-therapy group (11.1\%). After correction for imbalance in baseline characteristics, the HR remained similar $(0.56,95 \% \mathrm{CI}$ $0.35-0.91)$. This result indicated that the use of clopiogrel without aspirin was associated with a significant reduction in bleeding complications and no increase in the rate of thrombotic events. The subjects of this trial are not acute coronary syndrome, but the combination of clopidogrel and anticoagulant might be one of the regimen in the stable stage after the onset.

Denmark registry also showed the combination of clopidogrel and $\mathrm{OAC}$ showed the equal incidence of myocardial infarction, stroke, and cardiovascular death to triple therapy. OAC plus as- pirin or DAPT significantly increased the cardiovascular death compared to triple therapy. ${ }^{30)}$ Clopidogrel and OAC are also suggested as a candidate for patients with NVAF treated by PCI.

In the real world in Japan registry, CREDO-KYOTO cohort $2,^{31)} 1,057(8.3 \%)$ were identified among 12,716 patients undergoing first PCI. Although the majority of AF patients had CHADS2 score $\geq 2$ (75.2\%), only 506 patients (47.9\%) received OAC with warfarin at hospital discharge. Cumulative 5-year incidence of stroke in the OAC group was not different from that in the no-OAC group $(13.8 \%$ vs. $11.8 \%, \mathrm{p}=0.49)$. Time in therapeutic range (TTR) with an international normalized ratio (INR) was obtained in 409 patients. Only 37.7 patients with INR of 1.6 -2.6 had TTR $\geq 65 \%$. Cumulative 5 -year incidence of stroke in patients with TTR $\geq 65 \%$ was markedly lower than that in patients with TTR $<65 \%$ (6.9\% vs. $15.1 \%, \mathrm{p}=0.01)$. These results indicated that $\mathrm{OAC}$ was underused and its intensity was mostly suboptimal in "real world" AF patients undergoing PCI, which lead to inadequate stroke prevention.

\section{Anti-thrombotic therapy in patients with acute coronary syndrome having NVAF}

The vast majority $(93.5 \%)$ of patients underwent percutaneous coronary intervention (PCI), with a success rate of $93.9 \%$ in Japanese registry. ${ }^{11)}$ DPAT was administrated to most of them and OAC was added to DAPT in case of patients with AF. In this circumstance, we should discuss the two issues.

Firstly, when should we stop the DAPT? In the United States Registry, ${ }^{32}$ ACTION-registry Get With the guideline, among 4,959 patients, $27.6 \%$ were discharged on triple therapy. Relative to DAPT, patients on triple therapy had a similar risk of MACE (adjusted hazard ratio [HR]: 0.99 [95\% confidence interval (CI): 0.86 to 1.16$])$ but significantly greater risk of bleeding requiring hospitalization (adjusted HR: 1.61 [95\% CI: 1.31 to 1.97]) and greater risk of intracranial hemorrhage (adjusted HR: 2.04 [95\% CI: 1.25 to 3.34]). Although the duration of DAPT is still under discussion, ${ }^{33)}$ DAPT would be better to be stopped earlier in the case of triple therapy. In patients after an ACS, treated medically or with PCI, 6 months of triple therapy should be the current default before stepping down to double therapy. In those with a high (uncorrectable) bleeding risk, the duration of triple therapy can be shortened from 6 to 1 months, or even to immediate double therapy (with either aspirin or clopidogrel) in highly selected cases. For all CAD patients with $\mathrm{AF}$, the default is to step down to anticoagulation in monotherapy after 1 year, except for those with a very high risk for coronary events and an acceptably low bleeding risk. ${ }^{34)}$

Secondary, should we use NOAC besides warfarin? As discussed in the previous session, warfarin is not good for Asian and the use of warfarin did not show the stroke prevention in real 
world. ${ }^{35)}$ Japanese guideline of anticoagulant therapy for patients with NVAF recommends that NOAC is better than warfarin without contraindication. Antiplatelet use with NOAC increased the bleeding risk in all trials ${ }^{36-39)}$ and NOAC with DAPT did not have the benefit with increased the bleeding risk compared to DAPT. To decrease the risk of bleeding, European guideline recommended the reduced dose of NOAC with DAPT ${ }^{40)}$; however, their benefit in stroke prevention in patients with a normal renal function is uncertain. For the appropriate selection of oral anticoagulant, there are many options and there is no evidence right now. The risk of stent thrombosis is evaluated according the stent generation, stented legion (bifurcation, vessel size, etc.) and lesion characteristics (thrombus, lipid-rich plaque etc.) and bleeding risk is assessed by HAS-BLED score. ${ }^{41)}$ Taking these risk and benefit consideration, we should evaluate the net clinical benefit in each patient and then select the appropriate combination so far.

\section{Conclusion}

Warfarin is the only one oral anticoagulant for five decades. Non-vitamin K antagonist oral anticoagulant (NOAC) is available and showed the superiority of stroke prevention and less bleeding complication. Furthermore, NOAC is much more useful in Asians rather than the other race. NOAC has changed the concept of anticoagulant. This wave surged over the patients with coronary artery disease. NOAC will change the way of platelet use in patients with not only AF but also non AF. A lot of trials are going on. The subjects are different and the combination of antiplatelet and NOAC are variable. But the clinical trials answer the several clinical questions; however the indeed necessary clinical questions still remain. We have to continue the real world registry and seek for the answer the questions.

\section{Disclosure}

Atsushi Hirayama received honoraria from Bayer Pharmaceutical Co, Astellas Pharma Inc, Daiichi-Sankyo Co. Sanofi K.K. Sanwa Kagaku Kenkyusho Co, Boehringer Ingelheim Japan, Kyowa Hakko Kirin Co Ltd, Takeda Pharmaceutical Co Ltd, Kowa Co Ltd, Kissei Pharmaceutical Co Ltd, MSD K.K., Shionogi \& Co. Ltd., Eizai Co Ltd, Chugai Pharmaceutical Co, Pfizer Japan, Otsuka Pharmaceutical Co, Tanabe Mitsubishi Pharma Co, and Astra Zeneca KK. and received research support to institution from Astellas Pharmaceutical Co, Astra-Zeneca Co., Eisai Co, Ltd, MSD, Otsuka Pharmaceutical Co Ltd, ONO Pharmaceutical Co Ltd, Kissei Pharmaceutical Co Ltd, Kyowa Hakko Kirin Co Ltd, Goodman Co Ltd, KOWA Pharmaceutical Co Ltd, Sanofi K.K., Shionogi \& Co Ltd, Zeon Medical Inc, Daiichi Sankyo Co Ltd, Dainippon Sumitomo Pharma Co Ltd, Takeda Pharmaceutical Co Ltd, Mitsubishi Tanabe Pharma Corporation,
Chugai Pharmaceutical Co Ltd, Toa-Eiyo Ltd, Nippon Boehringer Ingelheim Co Ltd, Nihon Medi-Physics Co Ltd, Medtronic Japan Co Ltd, Pfizer Japan Inc, and Mochida Pharmaceutical Co Ltd.

\section{References}

1) Randomised trial of intravenous streptokinase, oral aspirin, both, or neither among 17,187 cases of suspected acute myocardial infarction: ISIS-2. ISIS-2 (Second International Study of Infarct Survival) Collaborative Group. Lancet 1988; 2: 349-360

2) Effects of tissue plasminogen activator and a comparison of early invasive and conservative strategies in unstable angina and non-Q-wave myocardial infarction. Results of the TIMI IIIB Trial. Thrombolysis in Myocardial Ischemia. Circulation 1994; 89: 1545-1556

3) Kodama K, Asakura M, Ueda Y, et al: The role of plaque rupture in the development of acute coronary syndrome evaluated by the coronary angioscope. Intern Med 2000; 39: 333-335

4) Cohen M: Glycoprotein IIb/IIIa receptor blockers in acute coronary syndromes: Gusto IV-ACS. Lancet 2001; 357: 1899-1900

5) Inhibition of platelet glycoprotein IIb/IIIa with eptifibatide in patients with acute coronary syndromes. The PURSUIT Trial Investigators. Platelet Glycoprotein IIb/IIIa in Unstable Angina: Receptor Suppression Using Integrilin Therapy. N Engl J Med 1998; 339: 436-443

6) Cannon CP, Weintraub WS, Demopoulos LA, et al: Comparison of early invasive and conservative strategies in patients with unstable coronary syndromes treated with the glycoprotein IIb/IIIa inhibitor tirofiban. N Engl J Med 2001; 344: 1879-1887

7) Antman EM: Glycoprotein IIb/IIIa inhibitors in patients with unstable angina/non-ST-segment elevation myocardial infarction: appropriate interpretation of the guidelines. Am Heart J 2003; 146(4 Suppl): S18-22

8) Yusuf S, Zhao F, Mehta SR, et al: Effects of clopidogrel in addition to aspirin in patients with acute coronary syndromes without ST-segment elevation. N Engl J Med 2001; 345: 494-502

9) Weaver WD, Simes RJ, Betriu A, et al: Comparison of primary coronary angioplasty and intravenous thrombolytic therapy for acute myocardial infarction: a quantitative review [see comments] [published erratum appears in JAMA 1998; 279: 1876]. Jama 1997; 278: 2093 2098

10) Urban P, Macaya C, Rupprecht HJ, et al: Randomized evaluation of anticoagulation versus antiplatelet therapy after coronary stent implantation in high-risk patients: the multicenter aspirin and ticlopidine trial after intracoronary stenting (MATTIS). Circulation 1998; 98: 2126-2132

11) Daida H, Miyauchi K, Ogawa H, et al: Management and two-year long-term clinical outcome of acute coronary syndrome in Japan: prevention of atherothrombotic incidents following ischemic coronary attack (PACIFIC) registry. Circ J 2013; 77: 934-943

12) Anderson JL, Adams CD, Antman EM, et al: ACC/AHA 2007 guidelines for the management of patients with unstable angina/non ST-elevation myocardial infarction: a report of the American College of Cardiology/American Heart Association Task Force on Practice Guidelines (Writing Committee to Revise the 2002 Guidelines for the Management of Patients With Unstable Angina/Non ST-Elevation Myocardial Infarction): developed in collaboration with the American 
College of Emergency Physicians, the Society for Cardiovascular Angiography and Interventions, and the Society of Thoracic Surgeons: endorsed by the American Association of Cardiovascular and Pulmonary Rehabilitation and the Society for Academic Emergency Medicine. Circulation 2007; 116: e148-304

13) Kohro T, Hayashi D, Okada Y, et al: Effects of medication on cardiovascular events in the Japanese coronary artery disease (JCAD) study. Circ J 2007; 71: 1835-1840

14) Merlini PA, Bauer KA, Oltrona L, et al: Persistent activation of coagulation mechanism in unstable angina and myocardial infarction. Circulation 1994; 90: 61-68

15) Hurlen $M$, Abdelnoor $M$, Smith $P$, et al: Warfarin, aspirin, or both after myocardial infarction. N Engl J Med 2002; 347: 969-974

16) Rothberg MB, Celestin C, Fiore LD, et al: Warfarin plus aspirin after myocardial infarction or the acute coronary syndrome: meta-analysis with estimates of risk and benefit. Ann Intern Med 2005; 143: 241 250

17) van Es RF, Jonker JJ, Verheugt FW, et al: Aspirin and coumadin after acute coronary syndromes (the ASPECT-2 study): a randomised controlled trial. Lancet 2002; 360: 109-113

18) Effects of long-term, moderate-intensity oral anticoagulation in addition to aspirin in unstable angina. The Organization to Assess Strategies for Ischemic Syndromes (OASIS) Investigators. J Am Coll Cardiol 2001; 37: 475-484

19) Connolly SJ, Ezekowitz MD, Yusuf S, et al: Dabigatran versus warfarin in patients with atrial fibrillation. N Engl J Med 2009; 361: 1139_ 1151

20) Ruff CT, Giugliano RP, Braunwald E, et al: Comparison of the efficacy and safety of new oral anticoagulants with warfarin in patients with atrial fibrillation: a meta-analysis of randomised trials. Lancet 2014; 383: 955-962

21) Morgan CL, McEwan P, Tukiendorf A, et al: Warfarin treatment in patients with atrial fibrillation: observing outcomes associated with varying levels of INR control. Thromb Res 2009; 124: 37-41

22) Toyoda K, Koga M, Hayakawa M, et al: Acute reperfusion therapy and stroke care in Asia after successful endovascular trials. Stroke 2015; 46: 1474-1481

23) Lip GY, Wang KL, Chiang CE: Non-vitamin K antagonist oral anticoagulants (NOACs) for stroke prevention in Asian patients with atrial fibrillation: time for a reappraisal. Int J Cardiol 2015; 180: 246254

24) Shen AY, Yao JF, Brar SS, et al: Racial/ethnic differences in the risk of intracranial hemorrhage among patients with atrial fibrillation. J Am Coll Cardiol 2007; 50: 309-315

25) Alexander JH, Lopes RD, James S, et al: Apixaban with antiplatelet therapy after acute coronary syndrome. N Engl J Med 2011; 365: 699-708

26) Mega JL, Braunwald E, Wiviott SD, et al: Rivaroxaban in patients with a recent acute coronary syndrome. N Engl J Med 2012; 366: 919

27) Oldgren J, Wallentin L, Alexander JH, et al: New oral anticoagulants in addition to single or dual antiplatelet therapy after an acute coronary syndrome: a systematic review and meta-analysis. Eur Heart J 2013; 34: 1670-1680

28) Toyoda K, Yasaka M, Uchiyama S, et al: Blood pressure levels and bleeding events during antithrombotic therapy: the Bleeding with Antithrombotic Therapy (BAT) Study. Stroke 2010; 41: 1440-1444

29) Dewilde WJ, Oirbans T, Verheugt FW, et al: Use of clopidogrel with or without aspirin in patients taking oral anticoagulant therapy and undergoing percutaneous coronary intervention: an open-label, randomised, controlled trial. Lancet 2013; 381: 1107-1115

30) Lamberts M, Gislason GH, Olesen JB, et al: Oral anticoagulation and antiplatelets in atrial fibrillation patients after myocardial infarction and coronary intervention. J Am Coll Cardiol 2013; 62: 981-989

31) Goto K, Nakai K, Shizuta S, et al: Anticoagulant and antiplatelet therapy in patients with atrial fibrillation undergoing percutaneous coronary intervention. Am J Cardiol 2014; 114: 70-78

32) Hess CN, Peterson ED, Peng SA, et al: Use and Outcomes of Triple Therapy Among Older Patients With Acute Myocardial Infarction and Atrial Fibrillation. J Am Coll Cardiol 2015; 66: 616-627

33) Montalescot G, Brieger D, Dalby AJ, et al: Duration of Dual Antiplatelet Therapy After Coronary Stenting: A Review of the Evidence. J Am Coll Cardiol 2015; 66: 832-847

34) Heidbuchel H, Verhamme P, Alings M, et al: Updated European Heart Rhythm Association Practical Guide on the use of non-vitamin K antagonist anticoagulants in patients with non-valvular atrial fibrillation. Europace 2015; 17: 1467-1507

35) Suzuki S, Yamashita T, Okumura K, et al: Incidence of ischemic stroke in Japanese patients with atrial fibrillation not receiving anticoagulation therapy--pooled analysis of the Shinken Database, J-RHYTHM Registry, and Fushimi AF Registry. Circ J 2015; 79: 432-438

36) Granger CB, Alexander JH, McMurray JJ, et al: Apixaban versus warfarin in patients with atrial fibrillation. N Engl J Med 2011; 365: 981-992

37) Eikelboom JW, Wallentin L, Connolly SJ, et al: Risk of bleeding with 2 doses of dabigatran compared with warfarin in older and younger patients with atrial fibrillation: an analysis of the randomized evaluation of long-term anticoagulant therapy (RE-LY) trial. Circulation 2011; 123: 2363-2372

38) Patel MR, Mahaffey KW, Garg J, et al: Rivaroxaban versus warfarin in nonvalvular atrial fibrillation. N Engl J Med 2011; 365: 883-891

39) Giugliano RP, Ruff CT, Braunwald E, et al: Edoxaban versus warfarin in patients with atrial fibrillation. N Engl J Med 2013; 369: $2093-$ 2104

40) Lip GY, Windecker S, Huber K, et al: Management of antithrombotic therapy in atrial fibrillation patients presenting with acute coronary syndrome and/or undergoing percutaneous coronary or valve interventions: a joint consensus document of the European Society of Cardiology Working Group on Thrombosis, European Heart Rhythm Association (EHRA), European Association of Percutaneous Cardiovascular Interventions (EAPCI) and European Association of Acute Cardiac Care (ACCA) endorsed by the Heart Rhythm Society (HRS) and Asia-Pacific Heart Rhythm Society (APHRS). Eur Heart J 2014; 35: 3155-3179

41) Okumura $\mathrm{K}$, Inoue $\mathrm{H}$, Atarashi $\mathrm{H}$, et al: Validation of $\mathrm{CHA}_{2} \mathrm{DS}_{2}-\mathrm{VASc}$ and HAS-BLED scores in Japanese patients with nonvalvular atrial fibrillation: an analysis of the J-RHYTHM Registry. Circ J 2014; 78: 1593-1599 